\title{
Novel resveratrol derivatives have diverse effects on the survival, proliferation and senescence of primary human fibroblasts.
}

\author{
Vishal C. Birar, ${ }^{+}$Angela N. Sheerin, ${ }^{+}$Elizabeth L. Ostler, ${ }^{*,+}$ and Richard G. A. Faragher. ${ }^{+}$ \\ 'School of Pharmacy and Biomolecular Sciences, University of Brighton, Cockcroft Building, \\ Moulsecoomb, Brighton BN2 4GJ, UK \\ *Author for correspondence: \\ Email: e.ostler@brighton.ac.uk
}

ABSTRACT: Resveratrol alters the cytokinetics of mammalian cell populations in a dose dependent manner. Concentrations above $25-50 \mu \mathrm{M}$ typically trigger growth arrest, senescence and/or apoptosis in multiple different cell types. In contrast, concentrations below $10 \mu \mathrm{M}$ enhance the growth of log phase cell cultures and can rescue senescence in multiple strains of human fibroblasts. To better understand the structural features that regulate these effects, a panel of 24 structurallyrelated resveralogues were synthesised and evaluated for their capacity to activate SIRT1, as determined by an ex-vivo SIRT1 assay, their toxicity, as measured by lactate dehydrogenase release, and their effects on replicative senescence in MRC5 human fibroblasts as measured by their effects on Ki67 immunoreactivity and senescence-associated $\beta$ galactosidase activity.

Minor modifications to the parent stilbene, resveratrol, significantly alter the biological activities of the molecules. Replacement of the 3,5-dihydroxy substituents with 3,5-dimethoxy groups significantly enhances SIRT1 activity, and reduces toxicity. Minimising other strong conjugative effects also reduces toxicity, but negatively impacts SIRT1 activation.

At $100 \mu \mathrm{M}$ many of the compounds, including resveratrol, induce senescence in primary MRC5 cells in culture. Modifications that reduce or remove this effect match those that reduce toxicity leading to a correlation between reduction in labelling index and increase in LDH release.

At $10 \mu \mathrm{M}$, the majority of our compounds significantly enhance the growth fraction of log phase cultures of MRC5 cells, consistent with the rescue of a subpopulation of cells within the culture from senescence. SIRT1 activation is not required for rescue to occur but enhances the size of the effect.

KEYWORDS: resveratrol, senescence, toxicity, SIRT1 


\section{INTRODUCTION}

During the latter decades of the $20^{\text {th }}$ century organismal ageing was regularly conceptualised as the potential result of the interplay of dozens, if not hundreds, of biological mechanisms, each of which exerted small effects on lifespan (Kirkwood \& Franceschi, 1992; Medvedev, 1990). However, more recently the adoption of a criterion-based approach for potential mechanisms (that they manifest during normal ageing, that their experimental aggravation accelerates ageing and that their amelioration retards it) has substantially reduced the number of mechanisms that can currently be considered plausible. The best example of this ontological approach, the 'Hallmarks of ageing' lists cell senescence, genomic instability, loss of proteostasis, mitochondrial dysfunction, epigenetic alteration, stem cell exhaustion and altered intercellular communication as candidate major ageing mechanisms (López-Otín et al. 2013).

On this model it is possible to envisage two distinct ways in which a small molecule or similar intervention might lengthen lifespan. Firstly, it might directly interact with multiple hallmark mechanisms at once, in a manner analogous to that in which phloroglucinols such as hyperforin interact with the multiple pathways that are involved in depression (Butterweck, 2003). Secondly, interconnectedness between the ageing hallmarks might allow an intervention targeting one hallmark to indirectly effect one or more of the others (perhaps best described as 'domino impingement'). Distinguishing between these potential modes of action is clearly desirable for many reasons, including gaining a better understanding of the primary mode of action of compounds currently being used clinically to target hallmark mechanisms (such as quercetin, which is both a senolytic and a proteostatic modulator see Kirkland \& Tchkonia (2017), Wedel et al. 2017) and aiding in the development of more potent and/or selective next generation compounds.

Resveratrol (trans-3,5,4'-trihydroxystilbene) is an excellent candidate for a molecule which modulates multiple hallmark mechanisms simultaneously. Although initially proposed to act as a dietary restriction mimetic based in part on the observation that resveratrol supplementation of the standard diet of laboratory mice induces shifts in gene expression similar to those seen when animals undergo calorie restriction (Barger et al. 2008, Bhullar and Hubbard; 2015). A wealth of data have subsequently become available consistent with resveratrol affecting proteostasis (Corpas et al. 2019), stem cell exhaustion (Safaeinejad et al. 2018), mitochondrial dysfunction (de Oliveira et al. 216), genomic instability (Stopper et al. 2005) and intercellular communication (Huang et al. 2020). Recently we have also shown that resveratrol has profound effects on the inflammatory phenotype of senescent cells, and is capable of reversing cell senescence after it has become established (Latorre et al. 2017). 
Understanding the structural motifs necessary for interaction with different hallmark mechanisms would greatly enhance the utility of resveratrol and its analogues. To facilitate this we developed a rapid, robust and convenient protocol for resveralogue synthesis which we have used to create a structurally diverse set of compounds, many of which are novel. This paper reports the effects of these compounds on one of the best characterised hallmark mechanisms, the proliferation and senescence of primary human fibroblasts.

\section{MATERIALS AND METHODS}

\section{General synthetic procedures for resveralogues}

Reaction schemes and $V$ series identities are shown in schemes 1-3 and tables 1 and 2 below.

Detailed synthetic methods and characterisation data are presented in the electronic supplementary information.

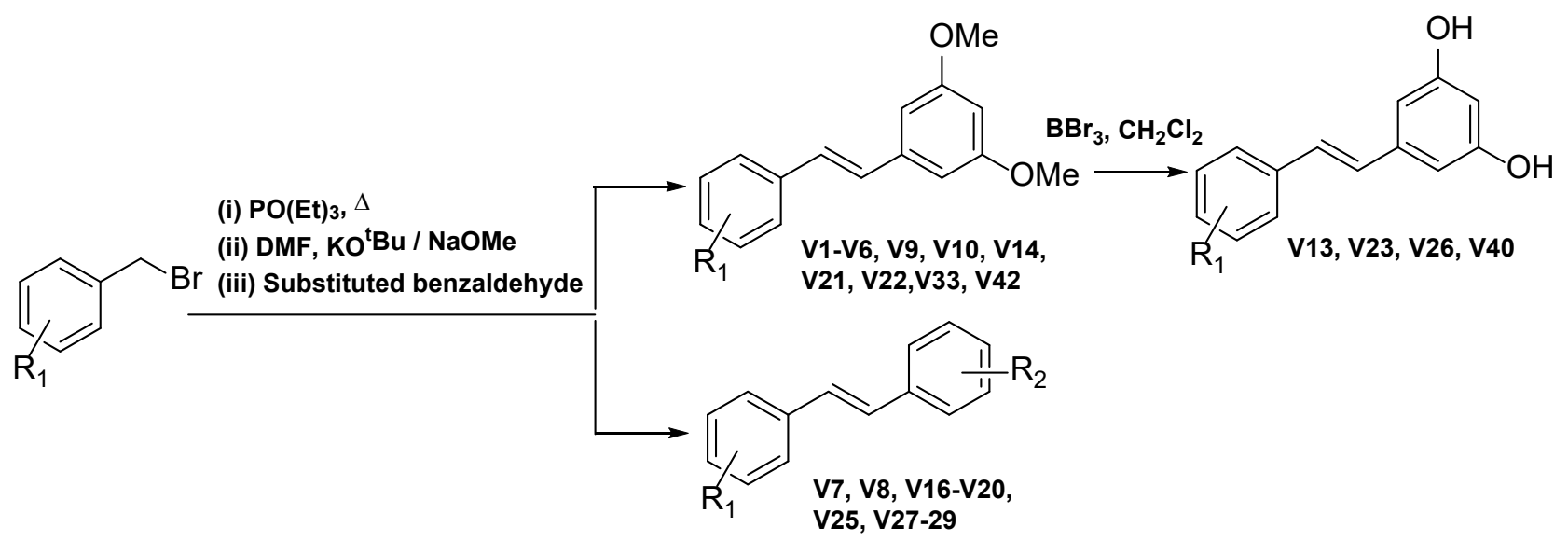

Scheme 1: Synthesis of simple resveralogues

Briefly, functionalised stilbenes were prepared from commercially available substituted benzyl bromides and benzaldehydes by using a modified Horner-Wadsworth Emmons reaction as previously described (Birar et al, 2015). Four of these were subjected to boron tribromide mediated deprotection to afford 3,5-diphenol analogues to facilitate comparisons with the parent 3,5,4'triphenol, resveratrol.

Table 1: Identities of simple resveralogues presented in $\mathrm{V}$ series number order

\begin{tabular}{|l|l|l|}
\hline Compound number & $\mathbf{R}_{\mathbf{1}}$ & $\mathbf{R}_{\mathbf{2}}$ \\
\hline V1 & $4-\mathrm{NO}_{2}$ & - \\
\hline V2 & $2-\mathrm{F}$ & - \\
\hline V3 & $4-\mathrm{MeO}$ & - \\
\hline V4 (V26: 3,5-Dihydroxy) & $3,5-$ Dimethyl & - \\
\hline V5 & $2,6-$ Difluoro & - \\
\hline
\end{tabular}




\begin{tabular}{|l|l|l|}
\hline V6 (V40: 3,5-Dihydroxy) & $2,4-$ Difluoro & - \\
\hline V7 & $3-\mathrm{Cl}$ & $4-\mathrm{MeO}$ \\
\hline V8 & $3-\mathrm{CF}_{3}$ & $4-\mathrm{NMe}_{2}$ \\
\hline V9 & $4-\mathrm{COOMe}$ & - \\
\hline V10 (V23: 3,5-Dihydroxy) & $2-\mathrm{CN}$ & - \\
\hline V14 (V13: 3,5-Dihydroxy) & $4-\mathrm{Me}$ & - \\
\hline V16 & $2-\mathrm{CN}$ & $2-\mathrm{CN}$ \\
\hline V17 & $3-\mathrm{Cl}$ & $4-\mathrm{NMe}_{2}$ \\
\hline V18 & $2-\mathrm{NO}_{2}$ & $4-\mathrm{MeO}$ \\
\hline V19 & $2-\mathrm{CN}$ & $4-\mathrm{MeO}$ \\
\hline V20 & $3-\mathrm{NO}_{2}$ & $4-\mathrm{MeO}$ \\
\hline V21 & $2,6-\mathrm{Dichloro}$ & - \\
\hline V22 & $2-\mathrm{NO}_{2}$ & - \\
\hline V25 & $2-\mathrm{NO}_{2}$ & $2-\mathrm{CN}$ \\
\hline V27 & $3-\mathrm{NO}_{2}$ & $2,4-\mathrm{Dimethoxy}$ \\
\hline V28 & $4-\mathrm{NO}_{2}$ & $2,4-\mathrm{Dimethoxy}$ \\
\hline V29 & $\mathrm{H}^{29}$ & H \\
\hline V33 & $4-\mathrm{CN}$ & - \\
\hline V42 & $\mathrm{H}$ & - \\
\hline
\end{tabular}
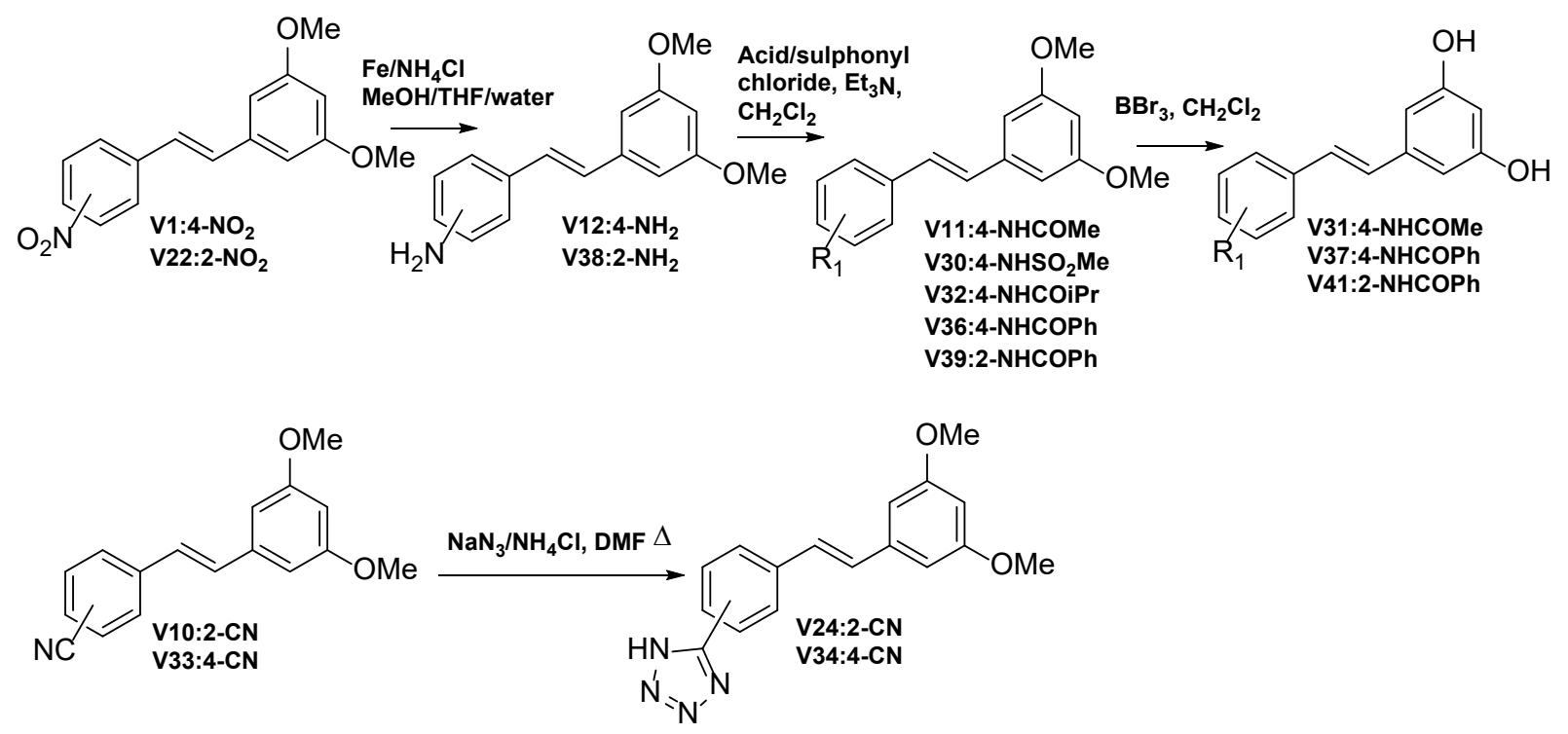

Scheme 2: Functional group modifications of nitrogen-substituted resveralogues

A range of nitrogen-substituted resveralogues were prepared from nitro- and cyano-substituted simple resveralogues (See also Latorre et al 2017). Anilines were prepared by reduction of nitro- 
substituted stilbenes, and then used to prepare a ranged of anilide and sulphanilide derivatives by reaction with the appropriate carboxylic acid or sulphonyl chloride. Three of these were also demethylated as above. Finally 2- and 4-tetrazole substituted analogues were afforded from 2-and 4-cyano stilbenes via Finnegan's method.

Table 2: Identities of modified nitrogen-substituted resveralogues presented in $\mathbf{V}$ series number order

\begin{tabular}{|l|l|l|}
\hline Compound number & Ring 1 substitution & Ring 2 substitution \\
\hline V11 & 4-NHCOMe & 3,5-Dimethoxy \\
\hline V12 & 4-NH 2 & 3,5-Dimethoxy \\
\hline V24 & 2-Tetrazole & 3,5-Dimethoxy \\
\hline V30 & 4-NHSO $2 \mathrm{Me}$ & 3,5-Dimethoxy \\
\hline V31 & 4-NHCOMe & 3,5-Dihydroxy \\
\hline V32 & 4-NHCO'Pr & 3,5-Dimethoxy \\
\hline V34 & 4-Tetrazole & 3,5-Dimethoxy \\
\hline V36 & 4-NHCOPh & 3,5-Dimethoxy \\
\hline V37 & 4-NHCOPh & 3,5-Dihydroxy \\
\hline V38 & 2-NH & 3,5-Dimethoxy \\
\hline V39 & 2-NHCOPh & 3,5-Dimethoxy \\
\hline V41 & 2-NHCOPh & 3,5-Dihydroxy \\
\hline
\end{tabular}

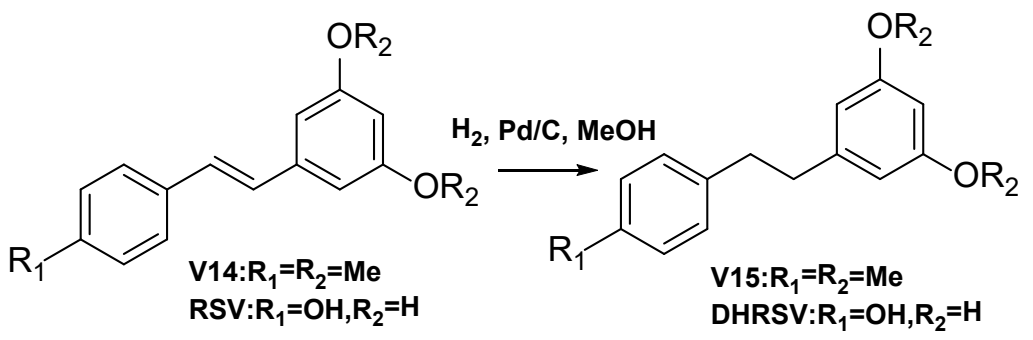

Scheme 3: Synthesis of dihydroresveralogues

Dihydro analogues of both resveratrol and V14 were readily prepared by palladium-catalysed reduction as described previously (Faragher et al 2010).

\section{Determination of SIRT1 activation capacity of novel resveralogues by measurement of lysyl deacetylase activity}

A commercial kit (SIRT1 fluorometric drug discovery kit, Cayman) based on the Fluor de Lys assay was used to determine the potential of each resveralogues to activate sirtuin 1 . All reactions were 
carried in reaction buffer $\left(50 \mathrm{mM}\right.$ Tris/ $\mathrm{HCl}, \mathrm{pH} 8.0,137 \mathrm{mM} \mathrm{NaCl}, 2.7 \mathrm{mM} \mathrm{KCl}, 1 \mathrm{mM} \mathrm{MgCl}_{2}, 1 \mathrm{mg} / \mathrm{cl}$ BSA and $2 \%(v / v)$ DMSO).

Resveralogues were diluted from a DMSO stock solution to a final concentrations of $25 \mu \mathrm{M}$ in reaction buffer. $5 \mu \mathrm{L}$ of purified SIRT1 enzyme solution was then added and $15 \mu \mathrm{L}$ of substrate solution was added to produce a final target peptide concentration of $125 \mu \mathrm{M}$ (with $3 \mathrm{mM} \mathrm{NAD}^{+}$in deacetylase reaction.) The reaction mixture was incubated on a shaker for 45 minutes at room temperature. After this time, stop solution was added and the mixture incubated for a further 30 minutes at room temperature. Fluorescence reading was measured using Perkin-Elmer VICTOR 1420 multi-label Plate Reader with excitation set at 360/40nm and emission measured at 460/40nm.

Activity was calculated according to the formula:

Activity $=[$ Fluorescence of sample - Initial fluorescence $] \times 100$

This was then normalised relative to enzyme only controls and resveratrol to give activation relative to resveratrol as a ratio, such that negative values represent enzyme inhibition, positive values indicate activation and compounds with values greater than one are more effective activators than resveratrol.

\section{General Tissue Culture}

MRC5 human diploid foetal lung fibroblasts (AG05965B)were obtained from the Coriell institute for medical research and grown at a seeding density of $1.3 \times 10^{4}$ cells $\mathrm{cm}^{-2}$ in Modified Eagle's Medium with essential and non-essential amino acids, 15\% FCS, $2 \mathrm{mM}$ glutamine, penicillin (50 U/L), streptomycin ( $50 \mu \mathrm{g} / \mathrm{L}$ ). Cells were sub-cultured every 5-10 days by trypsin-EDTA dispersion and viable cell numbers determined by trypan blue staining. Replicative lifespan was measured in Population Doublings (PDs) calculated using the standard formula. Fibroblast populations were dosed with resveralogues over a range of $10-100 \mu \mathrm{M}$ for 24 Hours after which the levels of cell death, senescence and proliferation were assessed.

\section{Determination of toxicity by lactate dehydrogenase release}

A commercial LDH cytotoxicity assay (Pierce LDH Cytotoxicity Assay Kit) was used to determine cell viability. MRC5 fibroblasts were seeded in 24 well plates with $1.3 \times 10^{5}$ cells $\mathrm{cm}^{-2}$ and allowed to recover from trypsinisation for 24 hours under standard tissue culture conditions. The cultures were then exposed the $10-100 \mu \mathrm{M}$ of the resveralogue for a further 24 hours. $50 \mu \mathrm{l}$ of media from each well was then mixed with an equal volume of LDH assay reaction mixture and incubated at room temperature in the dark for 30 minutes. $50 \mu \mathrm{l}$ of LDH assay stop solution was added to each well and 
the absorbance of the solution was measured by spectrophotometry at $490 \mathrm{~nm}$. Complete cell lysis and vehicle only controls were included.

\section{Determination of senescent fraction by catalytic histochemistry}

MRC5 fibroblasts were grown on $13 \mathrm{~mm}$ diameter glass coverslips at identical seeding densities to the parent culture. After exposure to resveralogues they were washed and fixed in $3 \%$ formaldehyde for $10 \mathrm{~min}$ at room temperature. The cells were washed and then incubated overnight at $37^{\circ} \mathrm{C}$ in a solution of 4-chloro-5-bromo-3-indolyl- $\beta$-D-galactoside in citric acid phosphate buffer containing potassium ferrocyanide $(5 \mathrm{mM})$ and potassium ferricyanide $(5 \mathrm{mM})$. As a positive control for lysosomal $\beta$-galactosidase activity, one set of coverslips was incubated in buffer at pH 4.0. For the determination of senescence-associated $\beta$-galactosidase, the incubation buffer $\mathrm{pH}$ was raised to $\mathrm{pH}$ 6.0. The fixed cells were counterstained using hematoxylin QS and Nuclear Fast Red and viewed under a light microscope. To determine the senescent fraction, 1000 cells were counted in random fields on two separate coverslips and the positive fraction calculated.

\section{Determination of growth fraction by Ki67 immunostaining}

MRC5 cells on cover slips were washed with PBS, and fixed in a 1:1 mixture of methanol and acetone for 5-10 minutes at room temperature. The fixed cells were incubated for one hour at room temperature with a primary antibody raised against Ki67 (Dako M0722, mouse anti-Ki67, diluted in 1\% (v/v) FCS in PBS 1:20 dilution). The cells were then washed three times in PBS and incubated for a further hour in the dark at room temperature with a secondary antibody conjugated with FITC (Dako F0261, rabbit anti-mouse FITC, diluted using 1\% (v/v) FCS in PBS, 1:20 dilution). Coverslips were washed three times in PBS and once in water to remove excess salt. They were then mounted in a DAPI-containing mountant (Vectashield) and viewed on a confocal microscope. Cells traversing the cell cycle showed a distinctive pattern of nuclear staining. To determine the proliferating fraction, either 400 Ki67 positive or 1000 total nuclei were counted in random fields on each of three coverslips.

\section{Statistical analysis}

All statistical analysis was carried out using Graph Pad-Prism. 


\section{RESULTS}

The structures of the $V$ series of resveralogues are shown in Schemes 1 - $\mathbf{3}$ together with Tables $\mathbf{1}$ and 2.

\section{SIRT Activation}

The resveralogues' capacity to activate or inhibit SIRT1 in the well-known 'Fluor de Lys' fluorescencebased histone deacetylase activity assay is shown in Figure 1A. Relative to resveratrol, the resveralogues displayed a spectrum of activities from increased activation (maximum of approximately two fold) through to inhibition.

The effect of single substituent modifications can be evaluated by comparison of the 3,5-dimethoxy series, and their deprotected 3,5-dihydroxy counterparts (see Table 3 below). In this series the highest values for SIRT 1 activation are observed both for compounds bearing electron donating (V3, V9) or electron withdrawing (V1) substituents on the second ring. Activation is further enhanced by replacement of the 3,5-dimethoxy substituents with 4-methoxy equivalents (V22 vs V18, V10 vs V19), but is reduced for analogous 2,4-dimethoxy compounds (V20 vs V27, V1 vs V28). However, no other consistent patterns in activity emerge in relation to variations in substituent positioning.

Deprotection of any one analogue to its 3,5-dihydroxy counterpart significantly (with the sole exception of the difluoro analogues) reduces SIRT1 activity, and in many cases converts a SIRT1 activator to an inhibitor (negative values) as seen in Table 3.

Table 3: SIRT1 activation ratios for dimethoxy resveralogues and their dihydroxy equivalents

\begin{tabular}{|l|c|c|c|c|c|}
\hline \multicolumn{1}{|c|}{$\mathbf{R}$} & $\begin{array}{c}\mathbf{R}^{\prime}=\mathbf{3 , 5}- \\
\text { Dimethoxy }\end{array}$ & $\begin{array}{c}\text { SIRT } \\
\text { activation } \\
\text { ratio (SD) }\end{array}$ & $\begin{array}{c}\mathbf{R}^{\prime}=\mathbf{3 , 5 -} \\
\text { Dihydroxy }\end{array}$ & $\begin{array}{c}\text { SIRT } \\
\text { activation } \\
\text { ratio (SD) }\end{array}$ & $\mathbf{p}$ \\
\hline 3,5-Dimethyl & $\mathrm{V} 4$ & $1.8(0.16)$ & $\mathrm{V} 26$ & $0.53(0.10)$ & 0.00030 \\
\hline 4-Methyl & $\mathrm{V} 14$ & $0.33(0.05)$ & $\mathrm{V} 13$ & $-1.33(0.19)$ & 0.00012 \\
\hline 4-Acetamide & $\mathrm{V} 11$ & $1.14(0.09)$ & $\mathrm{V} 31$ & $0.76(0.19)$ & 0.03516 \\
\hline 4-Benzamide & $\mathrm{V} 36$ & $0.2(0.04)$ & $\mathrm{V} 37$ & $-1.12(0.19)$ & 0.00030 \\
\hline 2-Benzamide & $\mathrm{V} 39$ & $-0.03(0.02)$ & $\mathrm{V} 41$ & $-0.24(0.07)$ & 0.00751 \\
\hline 2-Cyano & $\mathrm{V} 10$ & $0.68(0.05)$ & $\mathrm{V} 23$ & $-0.11(0.02)$ & 0.00001 \\
\hline 2,4-Difluoro & $\mathrm{V} 6$ & $0.5(0.11)$ & $\mathrm{V} 40$ & $0.48(0.09)$ & 0.81942 \\
\hline
\end{tabular}

In contrast, conversion of 2- and 4-amino-substituted resveralogues to amide equivalents (V12 and V38 to V11, V30, V32, V36 and V39) reduces SIRT1 activity significantly $(p<0.01)$ for all compounds except the 4-acetamide (V11). 
Hydrogenation of the central double bond (RSV to DHRSV, and V14 to V15) maintains or enhances SIRT activation activity.

\section{Toxicity}

The capacity of the novel resveralogues to induce necrotic cell death at $100 \mu \mathrm{M}$, as measured by the release of lactate dehydrogenase into the culture medium is shown in Figure 1B. As with SIRT1 activation small modifications to one ring of resveratrol gives rise to a wide range of toxicities ranging from indistinguishable from vehicle only control values to over $30 \%$ of positive control.

Dihydroxy analogues are generally slightly more toxic than their dimethoxy counterparts, as can be seen in Figure 2, but the differences observed are not significant. The highest toxicities in the $\mathrm{V}$ series are associated with 3, 5, 4'-trihydroxy (resveratrol), 4-amino (V8, V12, V17), dihalo (V5, V6, V21), 2-cyano (V10, V19, V23) and benzamide (V36, V39) substitution. In contrast, conversion of amines to amides (with the exception of 2-benzamide), and cyano to tetrazole derivatives, consistently reduces toxicity ( $p=0.049)$, suggesting that substituents that exert strong conjugative effects are causal for increased toxicity. This is in contrast with the effects on SIRT1 activity where removal of the hydroxyl group increases activity but loss of the amino group has the opposite effect. Reductive removal of the central double bond (RSV to DHRSV, and V14 to V15) reduces or maintains low toxicity, possibly by disrupting conjugation between the rings.

\section{Induction of senescence}

Like resveratrol, at $100 \mu \mathrm{M}$, many of the novel resveralogues also induce cellular senescence as demonstrated by the increased absence of staining for the proliferation marker pKi67, although as above there is a very wide range of activity (from no effect, to a reduction to less than half the labelling index observed with resveratrol, Figure 1C). For a subset of the $V$ series compounds this was confirmed by observation of a concomitant increased senescence-associated beta galactosidase activity (Figure 1C). A clear correlation is present between the capacity of resveralogues to induce necrosis and their capacity to induce senescence (Figure 3, $p>0.0006 r^{2}=0.53$ ). This is reinforced by the observation that the functional group interconversions that reduce toxicity (described above) also result in reduced induction of senescence at $100 \mu \mathrm{M}$.

\section{Increase in growth fraction}

Conversely, at $10 \mu \mathrm{M}$, treatment with a significant number of the $\mathrm{V}$ series compounds enhances the growth fraction of primary human fibroblasts as measured by staining for pKi67 (Figure 1D). Of all the compounds examined, those with SIRT1 activation activity similar to or greater than resveratrol $(\mathrm{V} 2,5,11, \mathrm{~V} 31)$ displayed significant increases in labelling index, whilst SIRT inhibitors (V13, V23, 
V37, V41) did not. However, several compounds with no significant SIRT activity (V30, V32, V36, V39) also provoked an increased labelling index. This dichotomy explains the weak but significant $\left(p=0.011, r^{2}=0.4361\right)$ correlation between SIRT1 activity and labelling index after treatment at 10 $\mu \mathrm{M}$. (Figure 4).

\section{DISCUSSION}

We have determined the effects on growth, death and senescence of a panel of novel resveralogues compared to the parent compound, resveratrol, and common metabolites such as

dihydroresveratrol. Accurately measuring the toxicity of resveralogues is more complex than generally assumed. Many researchers seeking to determine the toxicity of polyphenols use viability assays based on the reduction of MTT (3-(4,5-dimethylthiazol-2-yl)-2,5-diphenyltetrazolium bromide) which is soluble in aqueous solution but reduced to an insoluble formazan as a result of mitochondrial activity. The accuracy of this assay is predicated on a linear relationship between viable cell number, mitochondrial mass and mitochondrial activity. However, alterations in either mitochondrial content or metabolism influence the apparent viability of cell populations and resveratrol-like molecules are established activators of mitochondrial function (Bernhard et al. 2003). Other simple options for measuring cytotoxicity (e.g. neutral red uptake assays) can also be compromised because many polyphenols absorb strongly in the relevant wavelengths used to measure death.

Cognizant of these issues but above all because we wished to obtain an accurate measure of the induction of necrotic cell death we applied the lactate dehydrogenase release assay to our panel of resveralogues. In general, substituents such as halogens, hydroxyl and amine groups that exert strong conjugative effects increase the toxicity of these compounds, which is abrogated by conversion to "protected" methoxy or anilide equivalents. In contrast whilst methoxy analogues have better SIRT1 activation capacity than hydroxyl equivalents, anilide analogues display reduced SIRT1 activation when compared to the parent amine. Reductive removal of the central double bond also reduces toxicity and maintains or enhances SIRT1 activation. To our knowledge this is the first study of the structure activity relationships of resveralogues with regard to induction of necrosis.

Conversely, necrotic toxicity and the induction of replicative senescence by resveralogues at high doses correlate well. Given that resveratrol and its derivatives are regularly viewed as potential anticancer agents this could be seen as a useful feature - a single compound which potentially either kills tumour cells or renders them senescent. However, evidence is growing that the release of cytoplasmic proteins (e.g. HMGB1) into the extracellular space acts as a proinflammatory and angiogenic stimulus (Lee et al. 2018; Jain et al. 2013). Given that development of a necrotic core in 10 | $P$ a g e 
tumours is associated with poor patient prognosis the potential induction of necrosis by resveralogues is another reason, alongside the high doses required to induce senescence, that many of these compounds may not represent especially promising anti-tumour agents.

More promisingly, treatment of mid-passage cultures of MRC5 human fibroblasts with low concentrations of many, but not all, of the V series compounds increased the pKi67 index and decreased the fraction of cells in the cultures positive for senescence-associated beta galactosidase. Previously, we have shown that dosing senescent fibroblasts from multiple different tissues with resveratrol, dihydroresveratrol and four of our V series compounds results in a SIRT1-independent, SASP-independent and senolysis-independent exit from cellular senescence which we have termed 'rejuvenation'. Ki67 is always immunologically detectable across all cell cycle phases except $\mathrm{G}_{0}$ in log phase cell cultures (Kalashnik et al 2000) and modulation of Ki67 levels does not affect cell cycle kinetics (Sobecki et al 2016). Thus, the most plausible explanation for this increase is the 'rejuvenation' of a fraction of the senescent cell subpopulation within these cultures (rather than, for example, altered total cell cycle time or cell cycle phase duration). It is noteworthy that all the $\mathrm{V}$ series compounds which we have previously shown to allow senescent cells to divide again also induce a significant pKi67 spike (Latorre et al. 2017). Accordingly, we hypothesise that the compounds which increase the fraction of cells within log phase cultures staining positive for Ki67 will also rescue cell cultures composed entirely of senescent cells. Although activation of SIRT1 is not necessary for these effects to occur there is a clear trend for compounds which also activate SIRT1 to show a larger increase in the culture growth fraction. This is consistent with both SIRT1dependent and independent rescue mechanisms which can potentially be elucidated using the appropriate combination of compounds. It must be stressed that the 'rejuvenation' effect we have observed to date are transient and are dependent on the continued presence of the compound. Finally, in contrast, to the focus on SIRT1 activators such as resveratrol as anti-cancer drugs it has been proposed that SIRT1 inhibition may represent a utile strategy for the prevention of tumour growth. The canonical SIRT1 inhibitor, sirtinol, has been shown to induce senescence and apoptosis in MCF-7 cells (Wang et al. 2012) with an IC50 of $\sim 44-48 \mu \mathrm{M}$ depending on exposure time. At least one of our compounds (V13) may be especially well suited for an approach of this type since it shows higher SIRT1 inhibition than sirtinol, lower toxicity than resveratrol at $100 \mu \mathrm{M}(\mathrm{P}=0.039)$ and significantly reduces the pKi67 index of MRC5 fibroblasts at $25 \mu \mathrm{M}$. This is perhaps surprising given that the only structural difference between resveratrol and V13 is the replacement of the 4-phenol with a 4-methyl substituent, but highlights the importance of resonance effects in determining the activities of these molecules.

\section{Declarations}

11 | P a g e 
Funding: We thank the University of Brighton for a studentship for VB

Competing interests: None

Ethics approval: Not applicable

Consent to participate: Not applicable

Consent for publication: All authors consent

Availability of data and material: ESI includes details of procedures and characterisation. Novel compounds available in limited quantities.

Code availability: Not applicable

Author contributions: All authors made substantial contributions to the design of the work, analysis of data and production of the manuscript. All authors read and approved the final manuscript.

\section{REFERENCES}

Barger JL, Kayo T, Vann JM, Arias EB, Wang J, Hacker TA, Wang Y, Raederstorff D, Morrow JD, Leeuwenburgh C, Allison DB, Saupe KW, Cartee GD, Weindruch R, Prolla TA (2008) A low dose of dietary resveratrol partially mimics caloric restriction and retards aging parameters in mice. PLoS One 3(6):e226

Bernhard D, Schwaiger W, Crazzolara R, Tinhofer I, Kofler R, Csordas A (2003) Enhanced MTTreducing activity under growth inhibition by resveratrol in CEM-C7H2 lymphocytic leukemia cells. Cancer Lett. 195(2):193-9.

Bhullar KS and Hubbard BP (2015) Lifespan and healthspan extension by resveratrol. Biochimica et Biophysica Acta (BBA) - Molecular Basis of Disease.1852 (6) 1209-1218

Birar, V et al. (2015) A facile, stereoselective, one-pot synthesis of resveratrol derivatives. Chemistry Central Journal 9:26.

Butterweck V (2003) Mechanism of Action of St John's Wort in Depression: What Is Known? CNS Drugs 17(8):539-62.

Corpas R, Griñán-Ferré C, Rodríguez-Farré E, Pallàs M, Sanfeliu C. (2019) Resveratrol Induces Brain Resilience Against Alzheimer Neurodegeneration Through Proteostasis Enhancement. Mol Neurobiol. 56(2):1502-1516. 
de Oliveira MR, Nabavi SF, Manayi A, Daglia M, Hajheydari Z, Nabavi SM (2016) Resveratrol and the mitochondria: From triggering the intrinsic apoptotic pathway to inducing mitochondrial biogenesis, a mechanistic view. Biochim Biophys Acta. 1860(4):727-45.

Faragher RGA et al. (2010) Resveratrol, but not dihydroresveratrol, induces premature senescence in primary human fibroblasts. AGE epub:DOI: 10.1007/s11357-010-9201-5.

Latorre, E et al. (2017) Small molecule moderation of splicing factor expression is associated with rescue from cellular senescence. BMC Cell Biology 18:31 https://doi.org/10.1186/s12860-017-01477

Huang YC, Huang LT, Sheen JM, Hou CY, Yeh YT, Chiang CP, Lin IC, Tiao MM, Tsai CC, Lin YJ, Chen CC, Tain YL, Yu HR (2020) Resveratrol treatment improves the altered metabolism and related dysbiosis of gut programed by prenatal high-fat diet and postnatal high-fat diet exposure. J Nutr Biochem. 75:108260.

Jain MV, Paczulla AM, Klonisch T, Dimgba FN, Rao SB, Roberg K, Schweizer F, Lengerke C, Davoodpour P, Palicharla VR, Maddika S, Łos M (2013) Interconnections Between Apoptotic, Autophagic and Necrotic Pathways: Implications for Cancer Therapy Development. J Cell Mol Med17(1):12-29.

Kalashnik L, Bridgeman CJ, King AR, Francis SE, Mikhalovsky S, Wallis C, Denyer SP, Crossman F, Faragher RG (2000) A Cell Kinetic Analysis of Human Umbilical Vein Endothelial Cells. Mech Ageing Dev. 120(1-3):23-32.

Kirkland JL \& Tchkonia T (2017) Cellular Senescence: A Translational Perspective. EBioMedicine 21:21-28.

Kirkwood TB \& Franceschi C (1992) Is Aging as Complex as It Would Appear? New Perspectives in Aging Research Ann N Y Acad Sci 663:412-7.

Latorre E, Birar VC, Sheerin AN, Jeynes JCC, Hooper A, Dawe HR, Melzer D, Cox LS, Faragher RGA, Ostler EL, Harries LW (2017) Small molecule modulation of splicing factor expression is associated with rescue from cellular senescence. BMC Cell Biol. 18:31

Lee SY, Ju MK, Jeon HM, Jeong EK, Lee YJ, Kim CH, Park HG, Han SI, Kang HS. (2018) Regulation of Tumor Progression by Programmed Necrosis. Oxid Med Cell Longev. 3537471

López-Otín C, Blasco MA, Partridge L, Serrano M, Kroemer G (2013) The hallmarks of aging. Cell 153:1194-217

Medvedev ZA (1990) An attempt at a rational classification of theories of ageing. Biol. Rev. 65:375398. 
Safaeinejad Z, Kazeminasab F, Kiani-Esfahani A, Ghaedi K, Nasr-Esfahani MH (2018) Multi-effects of Resveratrol on stem cell characteristics: Effective dose, time, cell culture conditions and cell typespecific responses of stem cells to Resveratrol. Eur J Med Chem. 155:651-657.

Sobecki M, Mrouj K, Camasses A, Parisis N, Nicolas E, Llères D, Gerbe F, Prieto S, Krasinska L, David A, Eguren M, Birling M-C, Urbach S, Hem S, Déjardin J, Malumbres M, Jay P, Dulic V, Lafontaine DU, Feil R, Fisher D (2016) The cell proliferation antigen Ki-67 organises heterochromatin. eLife. 5:e13722. Stopper H, Schmitt E, Kobras K (2005) Genotoxicity of phytoestrogens. Mutat Res. 574(1-2):139-55. Wang J, Kim TH, Ahn MY, Lee J, Jung JH, Choi WS, Lee BM, Yoon KS, Yoon S, Kim HS. (2012) Sirtinol, a class III HDAC inhibitor, induces apoptotic and autophagic cell death in MCF-7 human breast cancer cells. Int J Oncol. 41(3):1101-9

Wedel S, Manola M , Cavinato M, Trougakos IP, Jansen-Dürr P (2018) Targeting Protein Quality Control Mechanisms by Natural Products to Promote Healthy Ageing. Molecules 23(5):1219. 

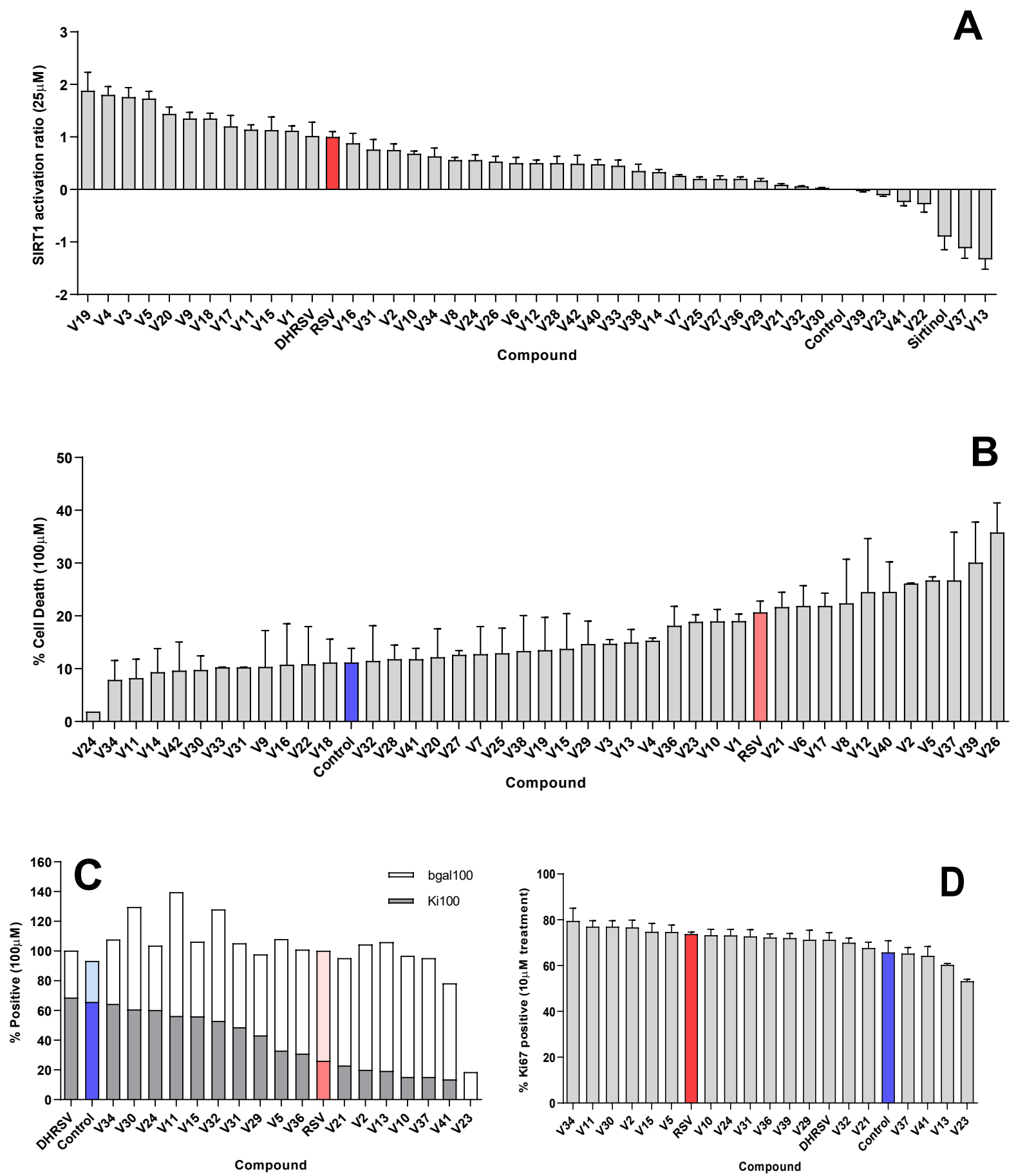

Figure 1 Selected cell physiological effects of the $V$ series resveralogues relative to resveratrol and vehicle only controls. Panel A SIRT1 activation capacities at $25 \mu \mathrm{m}$ relative to resveratrol (shown in red, activation $=1$ ) and the SIRT1 inhibitor sirtinol. Error bars \pm SD. Panel B Capacity of the V series resveralogues to induce necrosis in MRC5 human embryonic lung fibroblasts as measured by the release of lactate dehydrogenase following exposure to each compound at $100 \mu \mathrm{M}$ for 24 hours Error bars \pm SD. Panel C Stacking bar graph of Ki67 (grey) and senescence associated beta galactosidase indices (white) after treatment with $100 \mu \mathrm{M} V$ series resveralogues for 24 hours. 
Panel D Ki67 indices after treatment with $10 \mu \mathrm{M} V$ series resveralogues for 24 hours. Figure produced in GraphPad-Prism 


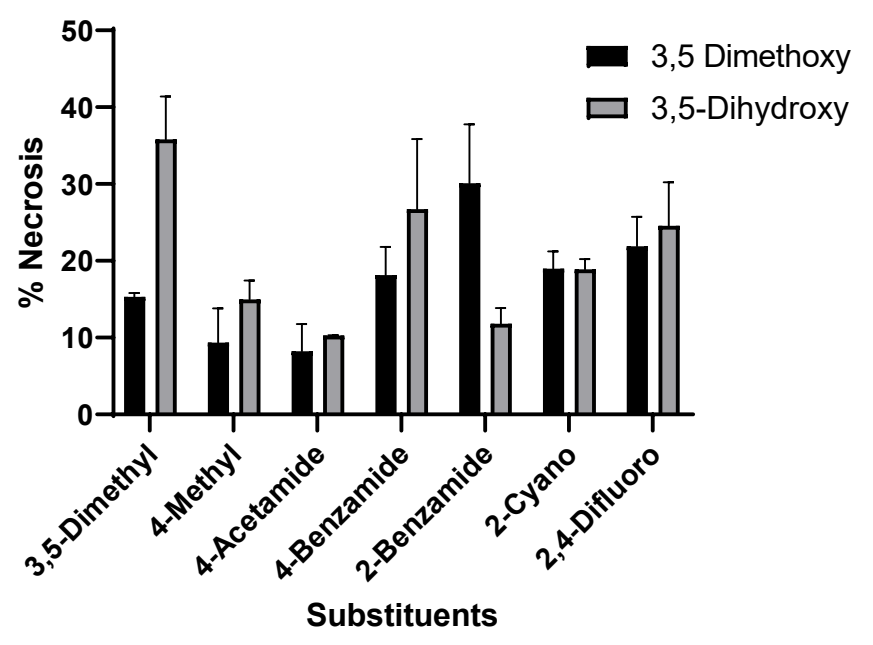

Figure 2 Comparison of matched 3,5-dihydroxy and 3,5-dimethoxy $V$ series resveralogues' capacity to induce necrosis in MRC5 human embryonic lung fibroblasts as measured by the release of lactate dehydrogenase following exposure to each compound at $100 \mu \mathrm{M}$ for 24 hours. Error bars $\pm S D$

Figure produced in GraphPad-Prism 


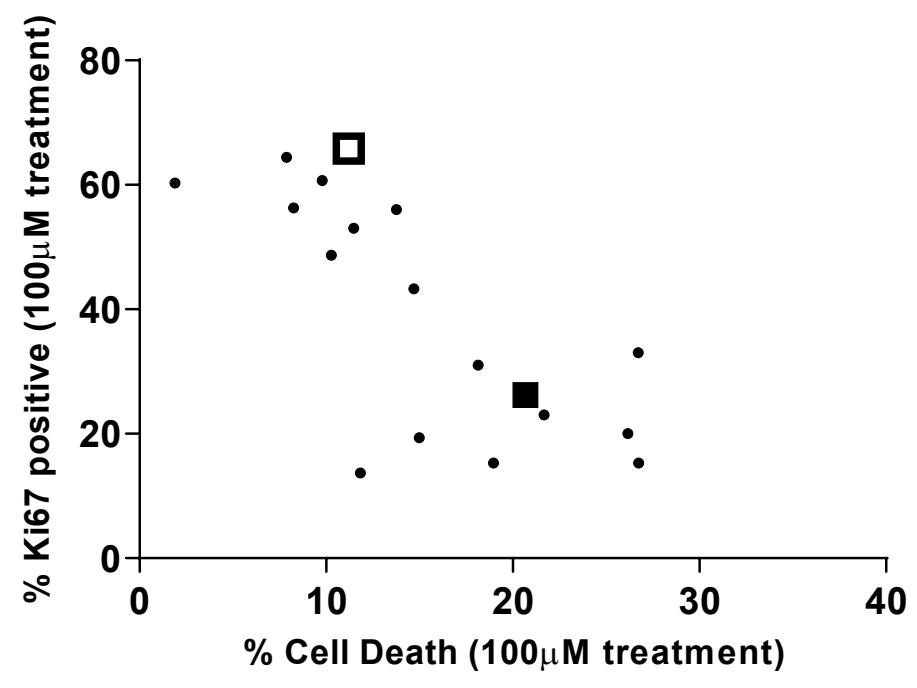

Figure 3 Capacity of the $V$ series resveralogues to induce senescence and necrosis in log phase cultures of MRC5 human embryonic lung fibroblasts as measured by a combination of loss of immunoreactivity for Ki67 ( $\mathrm{Y}$ axis) and release of lactate dehydrogenase (X axis). Vehicle only control and resveratrol shown as open and solid squares respectively

Figure produced in GraphPad-Prism 


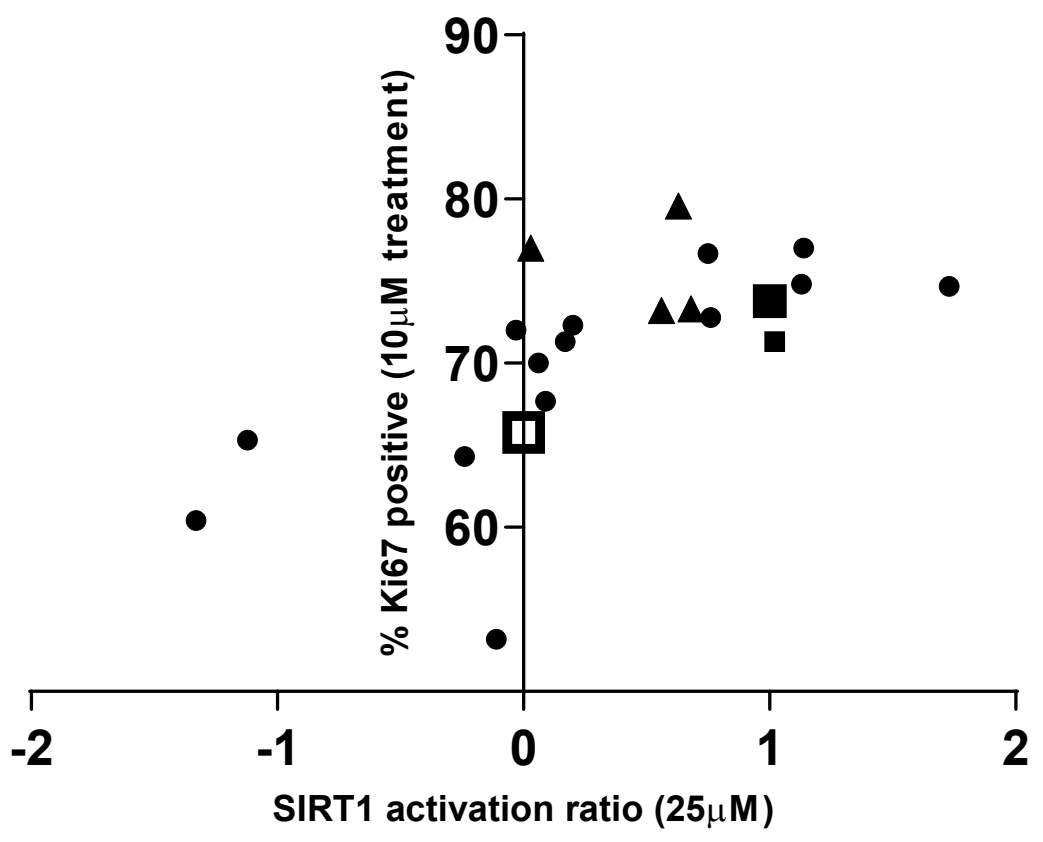

Figure 4 Relationship between the capacities of the V series resveralogues to activate SIRT1 and to alter the growth fraction of MRC5 human embryonic lung fibroblasts. Vehicle only control shown as an open square. Resveratrol and dihydroresveratrol shown as solid squares. $V$ series compounds previously shown to rescue senescence (Latorre et al. BMC Cell Biology (2017) 18:31) shown as solid triangles

Figure produced in GraphPad-Prism 